Chapter 6

\title{
Treatment of Large Cysts of the Mandible with Autografts of Cancellous Bone from the Tibia
}

\author{
Piotr Malara \\ Additional information is available at the end of the chapter \\ http://dx.doi.org/10.5772/53859
}

\section{Introduction}

Modern maxillofacial surgery is involved in treating a wide spectrum of diseases of the head and neck. Infectious diseases, cancers, traumas, as well as congenital and acquired malformations lie within the scope of this specialty. An important part of this specialty is treating diseases of the oral cavity and jaw bones, especially the removal of impacted teeth and orthognathic surgery. Treatment of large cysts is still a challenge for maxillofacial surgeons. The two-stage treatment is time-consuming, uncomfortable for patients and requires frequent check-ups. One-stage cystectomy of large cysts with water-tight closure of the postoperative bone cavity predisposes to complications (i.e. infection). Moreover, the weakened bone structure is prone to fractures in the postoperative period. This is why there is particular interest to fill the bone cavities with autografts and alloplastic materials. In the majority of cases no early complications are observed after cancellous bone harvesting from proximal tibia by medial approach to fill the bone cavities after cystectomies. Only moderate pain is experienced by the patients just after surgery allowing for early ambulation. Postoperative hospital stay ranges normally from 5 to 8 days depending on the size of the intraoral wound after cystectomy. However, the same day discharge postoperatively may be done. The volume of cancellous bone obtained ranges from 8 to $21 \mathrm{~cm}^{3}$. This chapter is based on our own experience and the literature review supportng the statement that cancellous bone harvesting from the proximal tibia via a medial approach is a relatively complication-free surgical procedure, which should be recommended when large postoperative cavities in mandible need to be grafted with significant amounts of cancellous bone. The aim of the chapter is to present the operative technique of cancellous bone harvesting from proximal tibia for filling large bone cavities after cystectomies. This chapter presents extensive step-by-step description of the 
operative technique. Special attention is focused on postoperative care and early rehabilitation. Possible complications related to the donor site, such as fractures due to the weakening of the tibia and donor site morbidity is presented in detail. The necessary period of postoperative hospital stay and mean volume of cancellous bone obtained will also be discussed.

\section{Background}

A serious problem in maxillofacial surgery is the persistence of a bone cavity as a result of a disease or after enucleation of intraosseous lesions. It is mostly associated with significant weakening of bony structures depending on the size of the bone cavity. In case of large cavities pathological bone fractures may appear as a result of even relatively slight trauma. Removal of a large intraosseous lesion may also cause esthetic problems and long-term functional problems. Moreover, in many cases tight closure of a bone cavity with soft tissue is difficult, which may cause further problems in the process of postoperative treatment. Tight closure of large bone cavities inevitably causes empty spaces. First, post-operative empty spaces fill with extravasated blood from adjacent tissues. Considering the regeneration process, it is a beneficial process, as the clot created from extravasated blood can lead to healing of the bone cavity as a result of a cascade of biochemical and cellular processes. It forms fibrous tissue which then goes through the process of mineralization, which results in filling of the bone cavity with newly created, full-fledged bone tissue. This process takes place according to the scenario above only in case of relatively small bone cavities. In cases with large bone cavities, which exceeds 4 $\mathrm{cm}$, there is a risk of multiple complications in healing which result from the retraction of the clot which appears in the closed post-operative cavity. Consequently, it may lead to the appearance of defective bone-like tissue which does not fulfill histological and clincal criteria. It is also important to remember that most operations in maxillofacial surgery are conducted via intraoral access. The consequence of conducting the surgery in an unsterile environment of the oral cavity is superinfection of the bone bed during the surgery and wound contact with bacterial flora of the oral cavity in the post-operative phase. Infection of a hematoma in the bone cavity with bacterial flora of the oral cavity may result in suppuration. The appearance of suppuration within the facial skeleton might progressively spread to adjacent spaces, in many cases making regenerative processes impossible, treatment length and expensive; it may also result in the occurrence of life-threatening complications. Therefore, in the case of large cysts their complete enucleation and closure via mucoperiosteal flap may leave a large empty space predisposed to infection of the hematoma which forms inside it during the post-operative period. Weakened bone structure also constitutes a risk for mandible fractures. Thus, special attention is paid to fill bone cavities with autogenous grafts and alloplastic materials. The advantage of autotransplantation is the fact that they have osteoconductive as well as osteogenic properties to accelerate the regeneration of bone, which should be the primary goal of the surgical procedure. 


\section{Grafts}

Most often autogenous bone grafts, as well as xenogenic and alloplastic materials are used to fill the cavities. Xenogenic materials are deprived of the organic components, which means that they include only inorganic components. It is similar to alloplastic materials which consist mainly of hydroxyapatite or calcium tri-phosphates. The advantage of alloplastic materials is the lack of a donor site and the reduction of patient discomfort. Its main drawback is that the materials consist mainly of inorganic components only. When implanted in the cavity they constitute a scaffold for infiltrating new blood vessels and connective tissue fibers; then they constitute a substrate of an inorganic phase for mineralization of the bone matrix. Therefore they mainly have osteoconductive properties with no osteogenic and osteoinductive properties. Therefore when such materials are used, significantly slower healing of bone cavities should be expected in comparison with grafts of bone tissue which are rich in living osteogenic cells and substances with osteoinductive properties.

Therefore bone grafts are a frequently used form of surgical treatment in maxillofacial surgery such as atrophy, congenital malformations, trauma and defects emerging as a result of cancer. Considering the fact that autogenous grafts are performed within the same body, the problem of potential graft rejection due to immunological incompatibility does not exist. While it is a significant problem in case of heterogeneous and xenogenic grafts [1]. Autografts are claimed to be the golden standard of reconstructive surgery; because the graft consists of organic and inorganic parts, and a large pool of osteogenic cells. As opposed to soft tissue grafts, the inorganic part of the bone graft creates a scaffold which functions as a bridge for the cells coming from the rim of the recipient site. The graft is colonized by cells, which undertake their functions and allow the survival of the graft [2]. Graft healing takes from three to six months and it is connected with a specified pattern of vascular and cellular processes [3].

The process of healing is connected with resorption and remodeling of the bone graft. The resorption degree and its pace depend on numerous factors, including the size of the grafted bone, its quality, as well as the quality of the donor site and the method of attachment of the graft in the recipient site [4]. It can be concluded from clinical observations that the smaller volume of grafted bone tissue, the slower the rate of graft resorption. The method of attachment of the graft in the recipient site is extremely important. On one hand, the attachment should ensure stable mounting of the grafted bone. Even the slightest movements of the graft on its base significantly increase the rate of resorption. On the other hand stability of the graft should be ensured using the smallest possible number of binding materials. The smaller amount of binding material, the slower the rate of the graft resorption clinically observed.

In transplant surgery two types of bone tissue coexisting in the human body can be used. Cortical bone thanks to the presence of Haversian channels, shows good osteoconductive properties. Due to its mechanical properties it can be used in cases when recreation of tridimensional cavities within the facial part of the skeleton is required. As opposed to cortical bone, cancellous bone is extremely rich in osteogenic cells. Living osteoblasts of cancellous 
bone may survive even for a few hours from the time of harvesting of the tissue; early revascularization in closed cavities takes place after 48 hours. The disadvantage of the cancellous bone grafts is their small mechanical endurance. It is also connected with the lack of possibility to use them in case of tridimensional reconstructions [5].

\section{Cysts}

An example of bone cavities in which the cancellous bone grafts can be used is a post-cystectomy cavity. Cysts in jaw bones occur relatively often and they are pathological changes within the facial skeleton. They are pathological spaces filled with liquid or semi-liquid content. Sometimes they are lined with epithelium. Their occurrence is not connected with the accumulation of purulent discharge. Development of jaw cysts is often asymptomatic. They are often diagnosed accidentally during routine dental or radiological examinations. Sometimes in case of large cysts located on the surface a deformation or facial asymmetry is observed. Cysts located directly under the mucosa of the oral cavity cause significant thinning of mucosa which has a bluish color in such cases. In a situation when developing cysts cause significant distension of bone structure, bending and crepitation of bone cortex can be felt during palpation. Cysts can also undergo secondary infection and then problems associated with purulent infection become most visible. Besides clinically diagnosed asymmetry, longterm growth of cysts may lead to tooth root movement, occlusion disorders and loosening of neighboring teeth. When a patient uses dentures, the ones that have been used up until that time may not be well-adjusted any more. In the mandible developing cysts may lead to disturbances of sensation from damage of the inferior alveolar nerve. Moreover, loss of bone tissue which occurs because of developing cysts may lead to pathological fractures of the mandible.

Cysts can be divided in many categories. From a practical point of view, cysts which appear most often are radicular cysts, also known as inflammatory. Their appearance is related with a presence of a non-vital tooth. In case of the absence of a tooth in the dental arch after the eruption time, an eruption cyst, a dentigerous cyst or a keratocystic odontogenic tumor must be taken into consideration. Among additional examinations, radiological examinations play a most significant role in the diagnosis of a cyst. In case of large cyst it is necessary to have an orthopantomographic (OPG) image to reveal the whole lesion. In radiological assessment the following should be taken into consideration: translucency of the lesion, its size and shape, the surrounding border changes, its relation to adjacent teeth, maxillary sinus and the lower alveolar nerve, displacement or resorption of teeth and the presence of opacity within the lesion.

Differential diagnosis of radiological entities observed within the facial skeleton should consider central giant cell granuloma, ameloblastoma, calcifying fibroma, myxoma and multiple myeloma.

The treatment of choice used in case of bone cysts is complete enucleation. Self-regeneration in most cases leads to complete healing of the bone cavity. While this method of treatment is 
acceptable in the case of small cysts, this approach arouses some controversies in the case of large cysts, especially those located in the mandible [6].

Marsupialization is a method of treating such changes which has been used to this day [7]. This procedure includes opening the cyst, draining its content and exposing the lining epithelium to the oral cavity. It is a relatively simple procedure and it has an advantage of reducing the risk of damaging the structures adjacent to the cyst, for example when the cyst spreads between the roots of vital teeth or in the direction of the lower alveolar nerve. Moreover, this method of treatment is recommended for older people and compromised patients for whom a lengthy surgical procedure is contraindicated. Its disadvantage is that pathologically changed tissue which lines the cyst remains in the body for another 6-18 months, during which the post-operative cavity is filled respectively with lint compresses, wax obturators and acrylate obturators. Marsupialization of cysts has good treatment results, however it is long-term, it requires frequent check-ups and it is rather uncomfortable for patients [8]. The whole surgical procedure should be finished with complete excision of pathologically transformed tissue at a later date together with a histopathological verification of the removed tissue. As mentioned above, the method of choice is a complete one-phase enucleation of the cyst. During the procedure the lining of the cyst should be precisely separated from adjacent structures so that the whole lesion can be enucleated in one piece. Surgical access is through the mucosa of the oral cavity and bone covering the cyst. The soft tissue incision should be performed far from the margins of the lesion to ensure the course of the suture line does not overlie the bone defect.

\section{Diagnostics and pre-surgical procedures}

Radiographs. Within pre-operative diagnostics in all patients with suspected mandibular cysts orthopantomographic (OPG) radiographs are taken (Fig. 1).

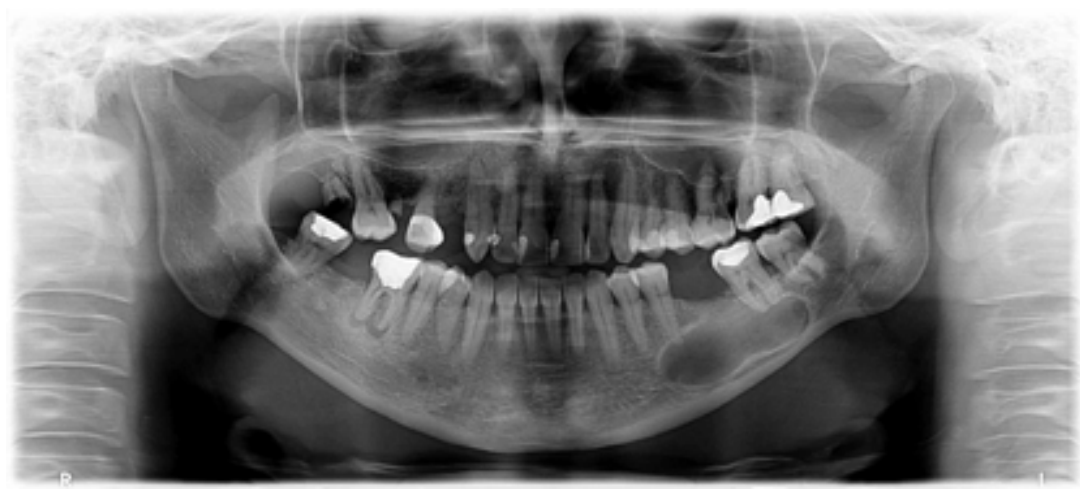

Figure 1. OPG of a large cyst in the left mandible. 
Biopsy. Biopsy for histopatological examination is also taken in the outpatient department. Patients with histopatological diagnosis of odontogenic cysts with sharply demarcated osteosclerotic margin and largest dimension exceeding $4 \mathrm{~cm}$ are candidates for surgical treatment to enucleate the cyst and direct fill the post-cystectomy defect with autogenous graft from the proximal tibia [8].

Computed tomography. A computed tomography (CT) scan of the facial skeleton is performed (Fig. 2), as well as an X-ray of the knee joints in the antero-posterior and lateral views (Fig. 3 and 4 ).

It is essential to consider data from the case history in selecting a limb to harvest cancellous bone (lack of previous fractures etc.), as well as the results of radiological examination (for lesions, defects etc. of the proximal tibia). When both limbs can constitute donor sites, the patient's preference should be taken into consideration.

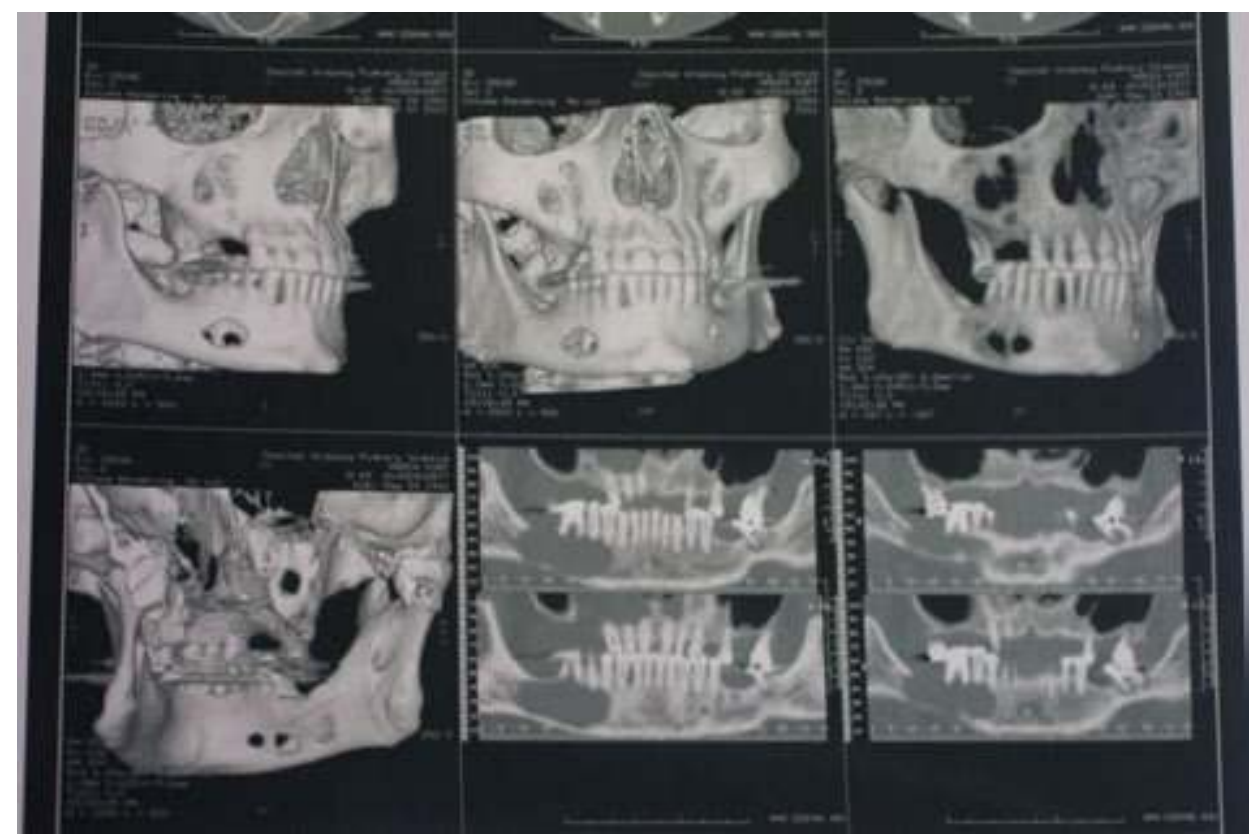

Figure 2. CT scans of a large cyst in the right mandible. 


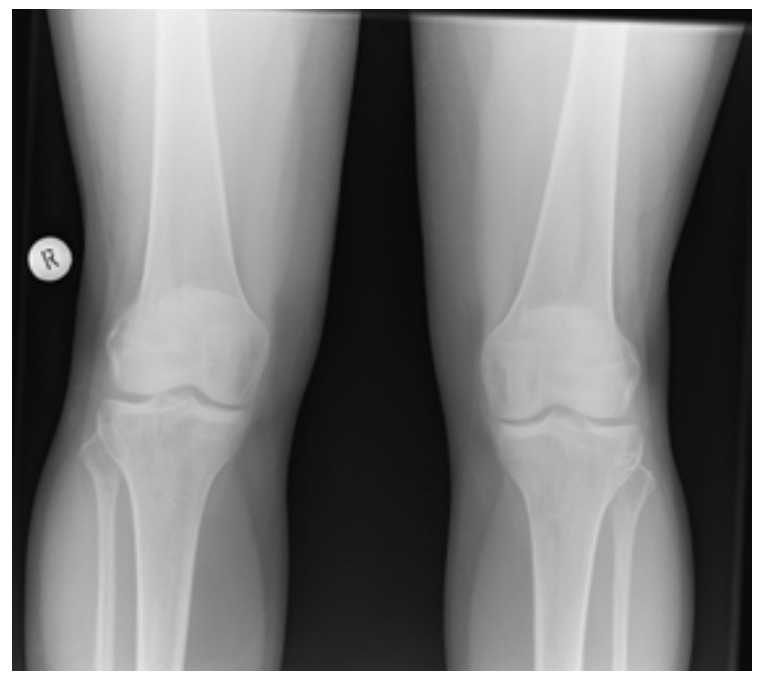

Figure 3. The P-A view of knee joints.

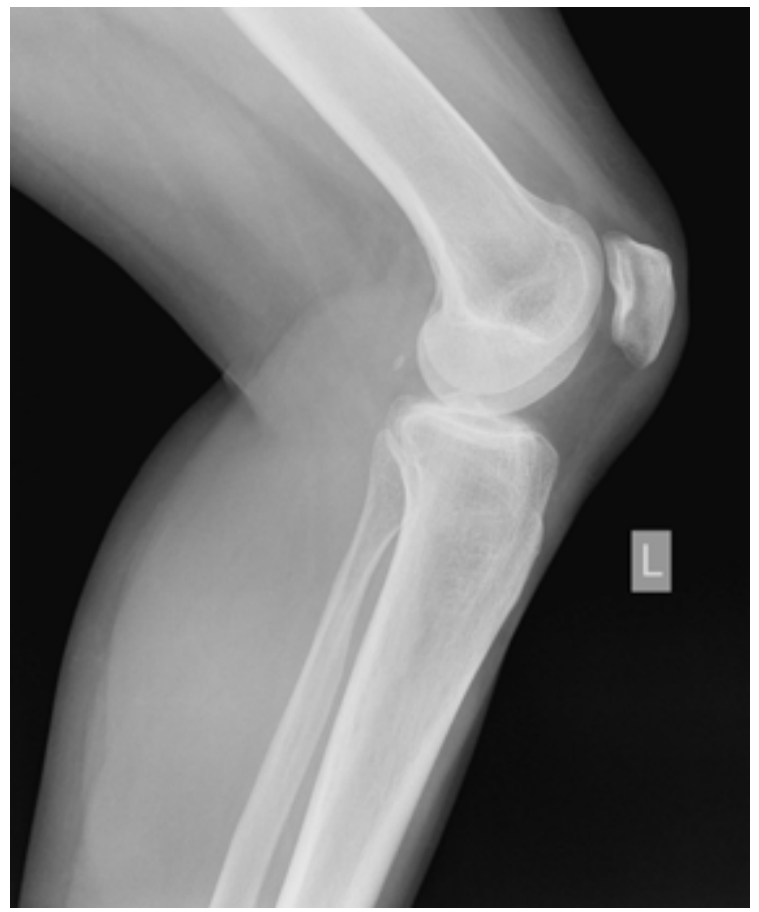

Figure 4. A lateral view of a knee joint. 


\section{Surgical technique for harvesting cancellous bone from the proximal tibia via medial access}

The tibia is a long bone, at the proximal end of which there are two condyles: medial and lateral. Between the condyles there is an intercondylar eminence limited on the sides by two intercondylar protuberences - medial and lateral. Both condyles are surrounded by vertically falling margo infraglenoidalis, below which tibial tuberosity is located at the front [10]. Tibial tuberosity is palpable, and defining its location is essential to avoid damaging the articular surface of a knee joint during the procedure. The patellar tendon, under which small branches of the upper and lower medial popliteal artery run, is attached to the proximal part of the tibial tuberosity. The tibialis anterior muscle is located on the lateral surface of the tibia below the preparation line which is necessary for proper access to the proximal base [10].

The procedure for harvesting cancellous bone from the proximal tibia can be carried out under general anesthesia or sedation. The skin around the knee and proximal part of the shin should be washed with antiseptics and the surgical field should be protected with sterile drapes. Medial access is possible via a skin incision $2-3 \mathrm{~cm}$ long $2 \mathrm{~cm}$ below and $2 \mathrm{~cm}$ medial from the anterior tibial tuberosity (Fig. 5).

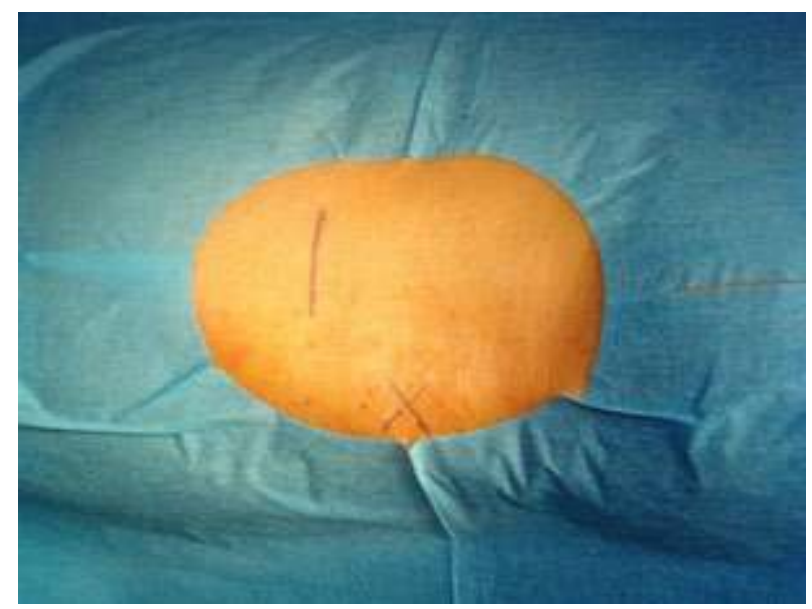

Figure $5.2-3 \mathrm{~cm}$ long incision is run $2 \mathrm{~cm}$ below and $2 \mathrm{~cm}$ medial to the anterior tibialis tuberosity.

The incision site is injected with 2 cc of $2 \%$ lignocaine with noradrenaline (1:80000) down to the periosteum. The incision is performed with blade no.10 through all the layers to the periosteum. Then the periosteum is reflected with a raspator in a way that allows free access to the medial surface of the proximal base. Soft tissues are retracted with Langenbeck retractors. The opening in the cortical plate of the tibia is performed using a trephine $8 \mathrm{~mm}$ in diameter mounted on a surgical handpiece at 120 revolutions per minute with copious sterile saline irrigation (Fig. 6). 


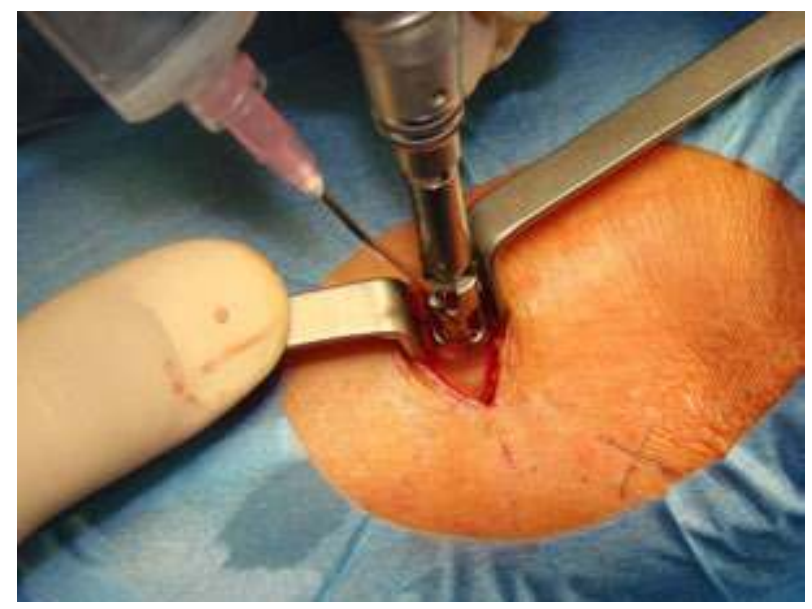

Figure 6. An opening in the cortical bone of tibia is made with a trephine $8 \mathrm{~mm}$ in diameter mounted on a surgical handpiece.

Then, through the opening straight and angled bone curettes of various sizes are used to mobilize cancellous bone. The curettes are not used to extract cancellous bone, but to separate cancellous bone from compact bone. A bone collector mounted on a surgical suction is used to extract cancellous bone (Fig. 7).

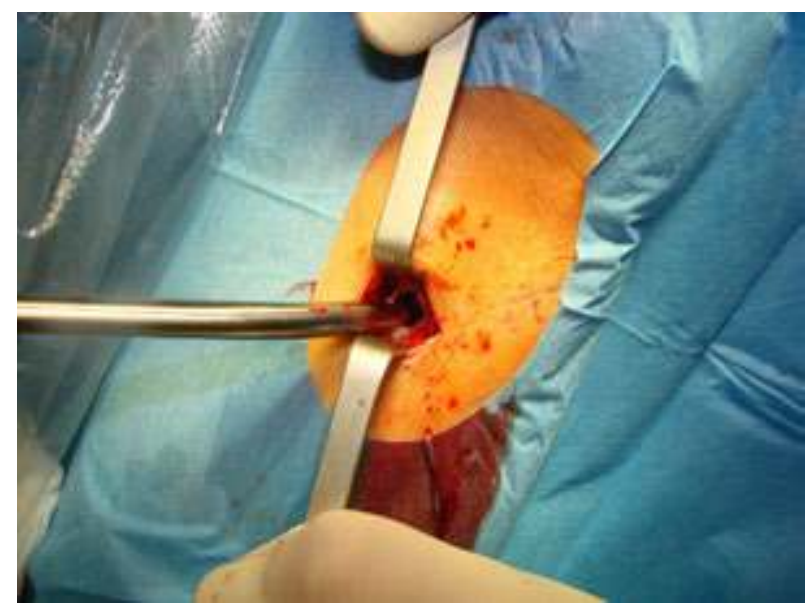

Figure 7. Cancellous bone from tibia is captured and removed with a bone collector connected to a suction.

In order to easily isolate fragments of cancellous bone extensive flushing with saline is performed through the opening. The procedure is performed until the moment when it is not possible to extract larger amounts of cancellous bone from the proximal base with manual 
instruments. Then a final flushing is performed with sterile saline solution, which is suctioned from the inside of the proximal base of the tibia using suction. The wound is closed in three layers. The periosteum is sutured using 3-0 absorbable sutures, subcutaneous tissue is sutured with 4-0 absorbable sutures. Skin is sutured with continuous intradermal suture using 5-0 nylon thread (Fig. 8). A sterile lint dressing is applied directly on the wound.

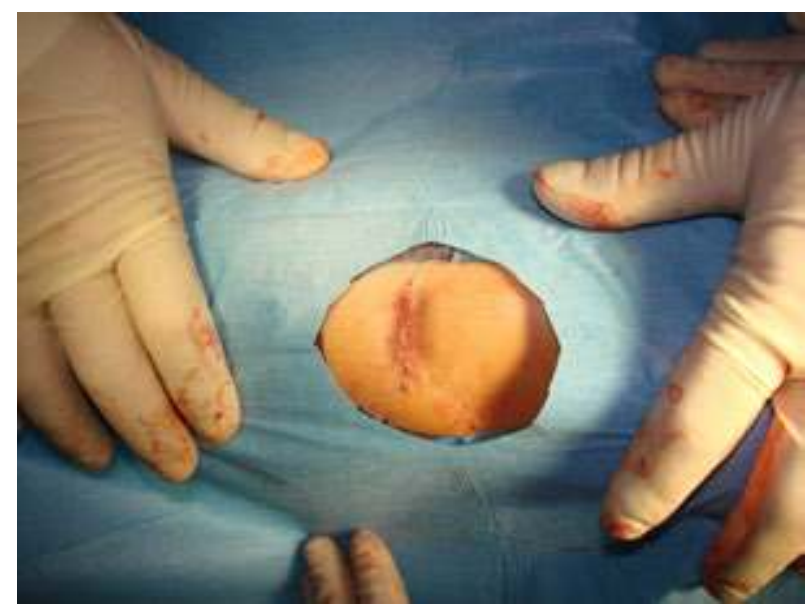

Figure 8. The skin wound is closed with intradermal nylon sutures.

Considering the extent of the surgery in the oral cavity patients are often candidates for surgical treatment under general anesthesia with endotracheal intubation through the mouth. In each surgery two surgical teams take part and they consist of the lead surgeon and an assistant. The task of one team is to harvest cancellous bone from the tibia, while the other team performs the intraoral cyst enucleation (Fig. 9) and places the harvested cancellous bone from the tibia in the post-cystectomy cavity (Fig. 10).

The surface of the transplanted cancellous bone in patients should be covered via the mucoperiosteal flap without damaging the periosteum and the flap must not require elongation, should directly cover the flap and the wound must be completely sutured with nylon sutures. In patients, in whom the mucoperiosteal flap requires elongation by undercutting the periosteum, the surface of cancellous bone is additionally covered with a membrane treated by platelet-rich plasma-derived fibrin clot (PRF) according to the methodology described by Choucroun et al. [11] (Fig. 11).

\section{Post-operative care}

Directly after the surgery the shin from the foot to the knee is wrapped in an elastic bandage. An elastic pressure dressing is held on the shin for 7 days. Antibiotics and non-steroid 
anti-inflammatory drugs are administered to patients, taking into consideration their general health and the extent of the surgery on the donor site. At afternoon hours on the day of the surgery patients are encouraged to walk. However, avoiding direct pressure on the operated limb, jumps, running the stairs, climbing a ladder, etc. is admissible 3 months after surgery. Control knee radiographs are taken after the surgery in order to confirm the accuracy of the opening in the cortical plate and to exclude possible fractures and infractions of the cortical plate (Fig. 12).

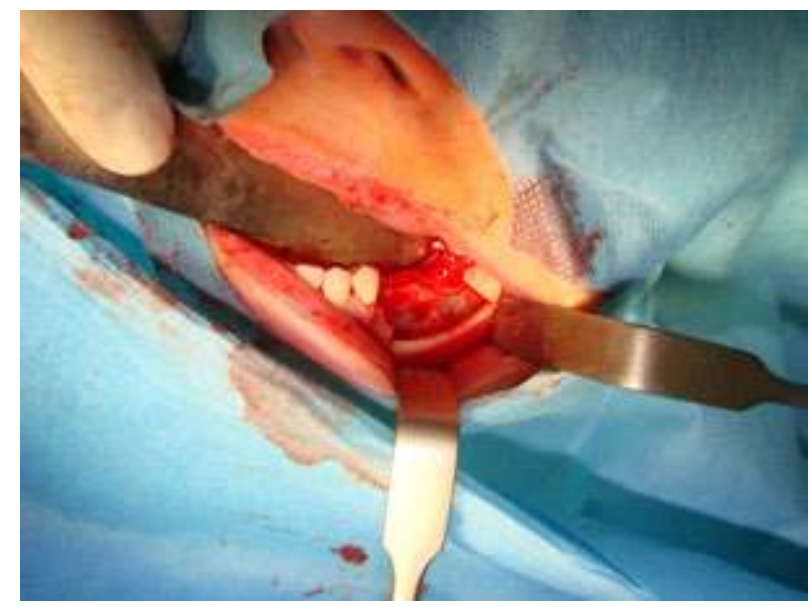

Figure 9. Cyst completely removed by an intraoral approach.

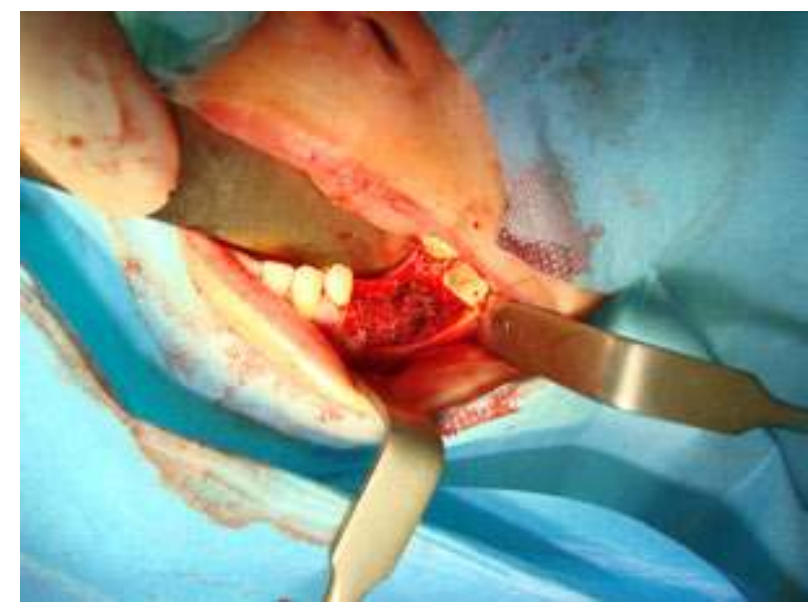

Figure 10. Bone cavity after cystectomy is filled with cancellous bone autograft. 


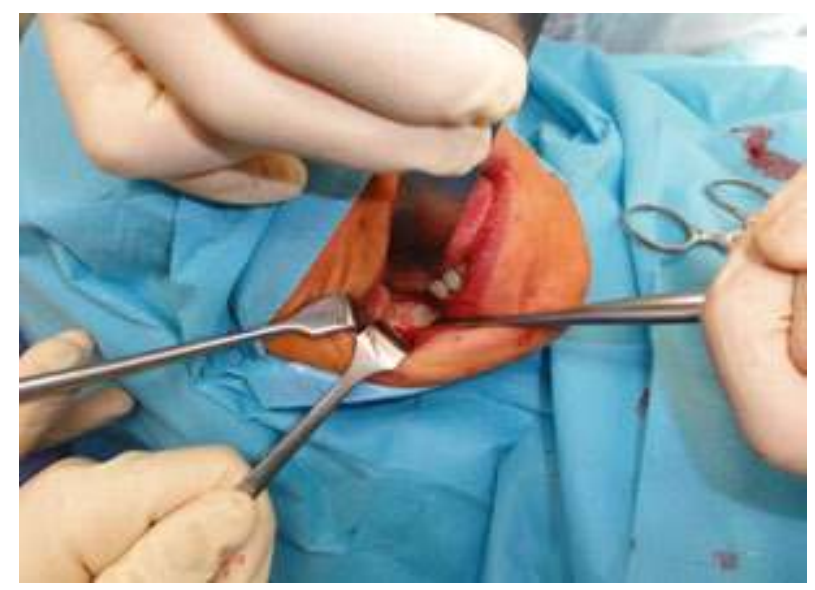

Figure 11. The cancellous bone graft is covered with PRF membranes.

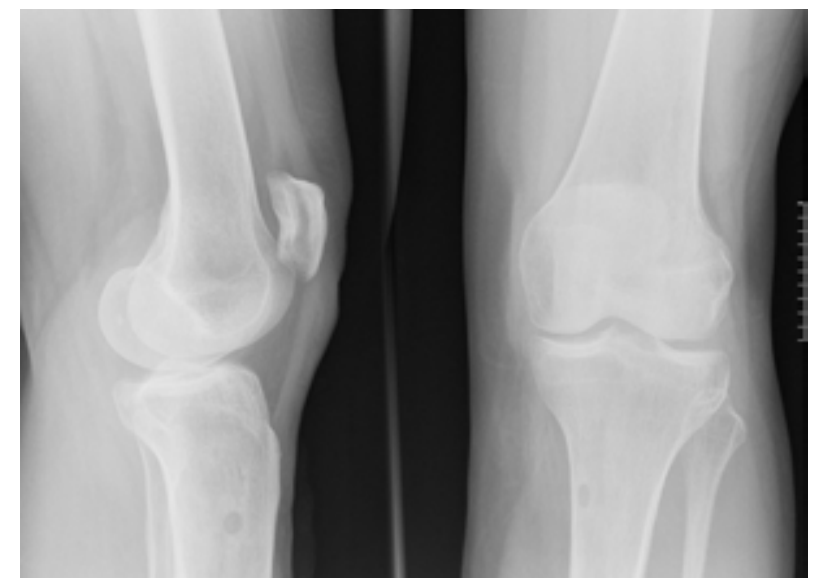

Figure 12. The P-A and lateral views of a knee joint taken after the harvesting procedure.

Sutures are removed between the $7^{\text {th }}$ and $10^{\text {th }}$ day after surgery. Hospitalization period depends on the extent of the surgery intraorally and healing of the recipient site. Considering the donor site, patients do not require post-operative hospitalization and they can be discharged on the day of the surgery.

Patients have out-patient control appointments after 7 days, 3 weeks, 3 months and 6 months after surgery. Besides clinical examinations, the appointment after 6 months includes the OPG X-ray in order to radiologically assess the healing of the bone cavity (Fig. 13). 


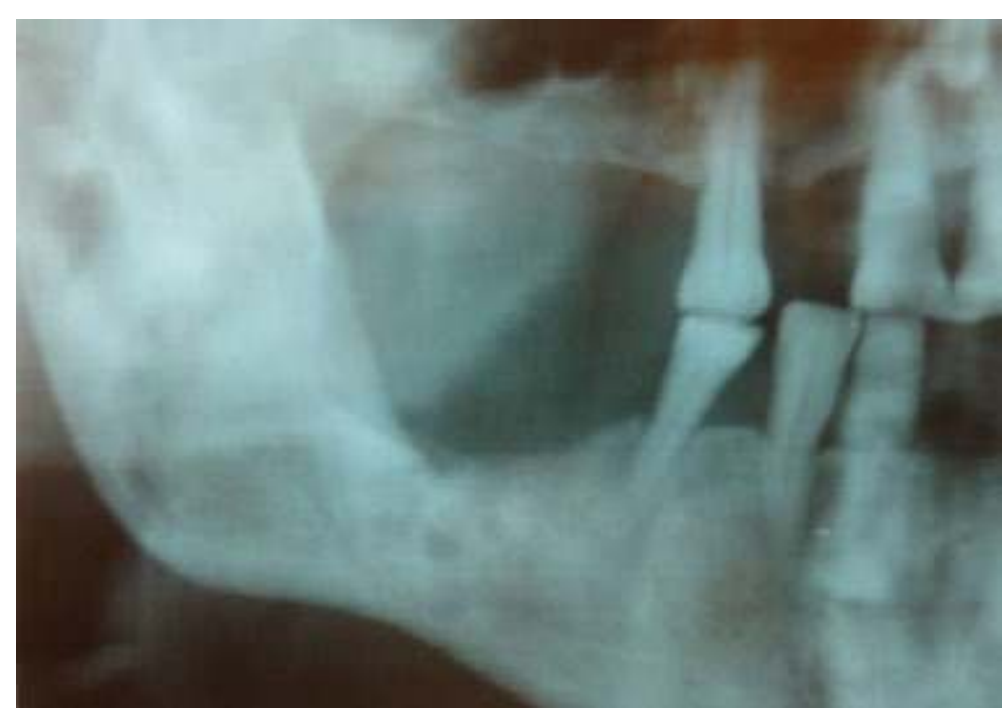

Figure 13. The OPG of a grafted cavity after cystectomy taken 6 months postoperatively. Total regeneration of the grafted cavity can be observed.

\section{Discussion}

Numerous treatments in maxillofacial surgery require autogenous bone grafts in order to fill bone cavities of the jaws. These treatments include reconstructive surgeries of clefts, postcancer defects and post-cystectomy defects. In the literature numerous donor sites are offered, including calvarium, symphysis of the mandible, ribs, iliac crest and tibia [12]. In clinical situations, in which it is necessary to use mechanical properties of cortical bone tissue, bone blocks which consist exclusively of cortical bone or cortical bone and cancellous bone are used. Plates of cortical bone undergo slower resorption than cancellous bone, however they include fewer cellular elements, and the process of osteogenesis shows slower dynamics [13]. Cancellous bone offers a significant pool of living pluripotent cells allowing osteogenesis, osteoinduction and osteoconduction [14]. Therefore in case of bone cavities with the geometry which allows filling with inlay techniques, surgeons prefer cancellous bone grafts. The majority of post-cystectomy cavities in the mandible belong to the category of such bone cavities. Choosing a donor site of the graft, expected amount and quality of harvested bone tissue should be taken into consideration, as well as the smallest possible post-operative discomfort for patients, difficulties in walking during the post-operative run, the length of necessary hospitalization period, possibility of early and late complications, as well as skills and preferences of the operator [12]. The area of the proximal base of the tibia has been used as a donor site in orthopedic surgery for many years [15, 16]. A while later attention was brought to the tibia as a donor site of cancellous bone for the needs of cranio- 
maxillofacial surgery, especially in the case of palate clefts and filling osteotomy gaps [17]. Since then harvesting surgical technique has been undergoing constant development and its aim is the smallest possible loss of cortical bone plate of the tibia [18] and saving the zone of growth in children [19].Surgical access to cancellous bone in the proximal base of the tibia can be obtained from the lateral surface of the bone with vertical skin incision running along the medial edge of anterior tibialis muscle below the tibial tuberosity [20] and via medial access through a skin incision running $2 \mathrm{~cm}$ below and $2 \mathrm{~cm}$ medially from tibial tuberosity [11]. The author of this chapter prefers medial access due to a lower number of described complications of this procedure. The medial surface of the tibia is located in this area directly under the skin. Access to the bone is obtained by an incision conducted concomitantly through all layers - skin, poorly developed subcutaneous tissue and periosteum. This way the risk of damaging muscles and larger blood vessels or nerves is reduced.

Average volume of cancellous bone obtained by different authors ranges from 10-42 cc [15, $17,19]$. Our own experiences indicate that in operated patients it was possible to obtain slightly smaller volumes of cancellous bone - from 8 to 21 cc (Fig. 14). However, in all operated patients the volumes obtained allowed filling of post-cystectomy cavities to a satisfactory degree. It should be pointed out that while planning the surgical treatment it must be considered that the amount of harvested bone from one limb may be insufficient and not possible to foresee pre-operatively. Therefore it is recommended to obtain patient consent to harvest bone from both tibias before the surgery [20,21].

In the literature a very small percentage of early complications at the donor site (from 0 to $1.9 \%$ ) draws attention; the complications include mostly impaired cutaneous wound healing, bleeding, severe postoperative pain and difficulty in walking manifested as limping [15, 22]. In the group of patients operated by the author no early or late complications in the donor site were observed. There are a few reports regarding a possibility of fractures of the tibia in patients in whom cancellous bone of the proximal base was harvested [20, 23]. Hughes and Revington [20] report an occurrence of this complication in 2 of the 75 operated patients [2.7\%). Even though we have not observed this complication in our own cases, it is possible that its occurrence is more frequent than it is shown by scientific reports, as many of such fractures can heal themselves without the need for surgical intervention, as suggested by Thor et al. [23]. Relatively short surgery time, short skin incision resulting in a small scar, a possibility to walk on the first day after surgery and short hospitalization period undoubtedly are advantages of cancellous bone graft from the base of the proximal tibia [17, 19-21, 24, 25]. All patients operated by the author are encouraged to walk in a few hours after the cystectomy which includes filling the cavity with cancellous bone from the base of the proximal tibia. The majority of the patients do not require any help in walking (crutches or walking stick) on the day of the surgery. However patients are recommended to avoid excessive pressure on the operated limb, such as running, jumping or climbing a ladder for 3 months. Contact sports are also discouraged in this period. [20,21]. It should be pointed out that considering the process of wound healing in the donor site, patients could be released home on the first post-operative day. The hospitalization period of some patients lasts between 5-8 days dictated by the extent of the intraoral wound after the cystectomy. It should 
be highlighted that at the donor site a good esthetic result in the form of a small linear scar visible on the shin is obtained (Fig. 15).

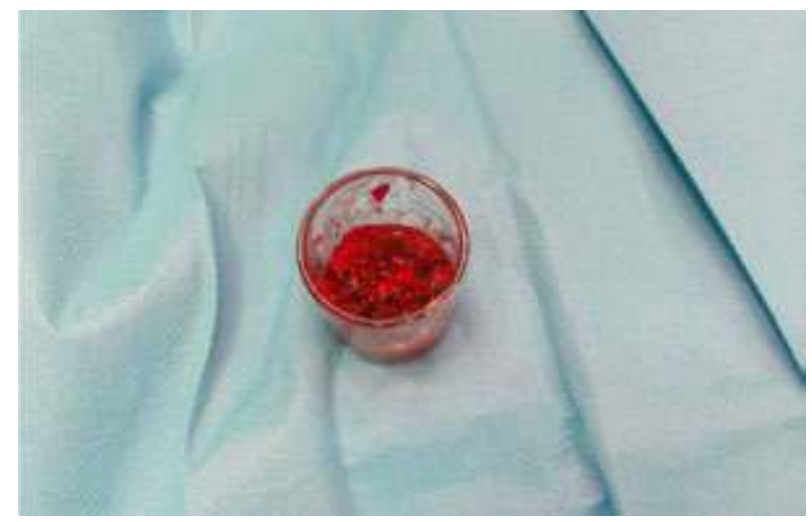

Figure 14. Harvested cancellous bone.

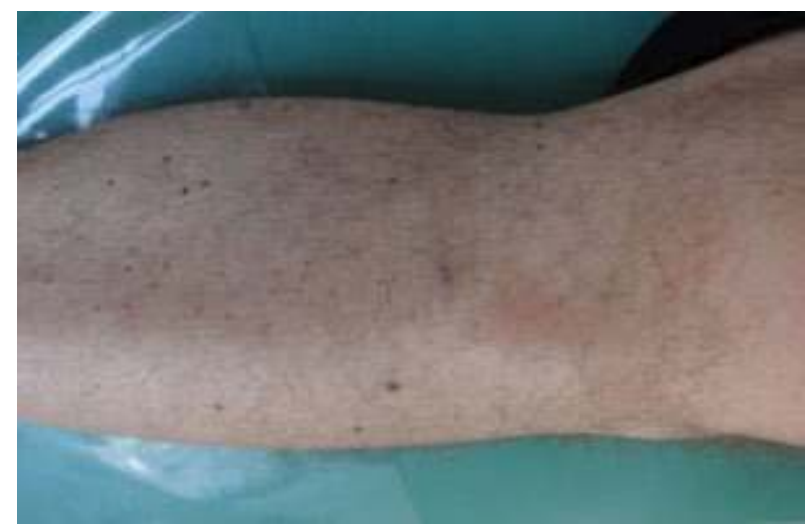

Figure 15. The skin scar 6 months postoperatively.

\section{Summary}

Previous experience and available literature allow it to be concluded that cancellous bone graft from the base of the proximal tibia via medial access is a relatively safe surgical technique which can be especially recommended when significant volumes of cancellous bone are needed to fill the recipient site. Relatively short surgery time, short skin incision resulting in an esthetically acceptable scar, a possibility to walk on the first day after surgery and short 
hospitalization period make this surgical technique an attractive alternative for different donor sites. Using cancellous bone graft from the base of the proximal tibia in order to fill postcystectomy cavities significantly contributes to the reduction of complications in treating large mandibular cysts with a method of complete enucleation and may lead to a significant acceleration of bone tissue regeneration at the recipient site.

\section{Author details}

Piotr Malara ${ }^{1,2}$

1 Department for Oral and Maxillofacial Surgery, DENTARIS Medical Centre, Ruda Slaska, Poland

2 Institute of Engineering Materials and Biomaterials, Silesian University of Technology, Gliwice, Poland

\section{References}

[1] Block M.S., Kent J. N.: Sinus augmentation for dental implants: The use of autogenous bone. J. Oral Maxillofac. Surg. 1997; 5: 1281-1286.

[2] Moy P.: Clinical experience with osseous site development using autogenous bone, bone substitutes and membrane barriers. Oral Maxillofac. Surg. Clin. North Am. 2001; 13: 493-509.

[3] Hollinger J. O., Wong M. E. K.: The integrated processes of hard tissue regeneration with special emphasis on fracture healing. Oral Surg. Oral Med. Oral Pathol. 1996; 82: 594-606.

[4] Fonseca R. J., Clark P. J., Burkes E. J., Baker R. D.: Revascularization and healing of onlay particulate autologous bone grafts in primates. J. Oral Maxillofac. Surg. 1980; 38: $572-577$.

[5] Citardi M. J., Friedman C. D.: Nonvascularized autogenous bone grafts in the treatment of the resorbed maxillary anterior alveolar ridge: Rationale and approach. Implant Dent. 1998; 7: 169-176.

[6] Chiapasco M., Allessandro R., Motta J. J., Crescentini M.: Spontaneous bone regeneration after enucleation of large mandibular cysts: A radiographic computed analysis of 27 consecutive cases. J. Oral Maxillofac. Surg. 2000; 58: 942-948.

[7] Nakamura N., Mitsuyasu T., Mitsuyasu Y., Taketomi T., Higuchi Y., Ohishi M.: Marsupialization for odontogenic keratocyst: long-term follow-up of the effects and changes in growth characteristics. Oral Surg. Oral Med. Oral Pathol. Oral Radiol Endod. 2002; 94: 543-553. 
[8] Enislidis G., Fock N., Sulzbacher I., Ewers R.: Conservative treatment of large cystic lesions of the mandible: a prospective study of the effect of decompression. Br. J. Oral Maxillofac. Surg. 2004; 42: 546-550.

[9] Etzias A., Sugar W.: Pathological fractures of the mandible: a diagnostic and treatment dilemma. Br. J. Oral Maxillofac. Surg. 1994; 32: 302-307.

[10] Herford A. S., Brett J. K., Audia F., Becktor J.: Medial approach for tibial bone graft: Anatomic study and clinical technique. J. Oral Maxillofac. Surg. 2003; 60: 358-363.

[11] Chounkroun J., Diss A., Simonpieria A., Girard M. O., Schoeffler C., Dohan S. L.: Platelet-rich fibrin (PRF): a second-generation platelet concentrate. Part IV: clinical effects on tissue healing. Oral Surg. Oral Med. Oral Pathol. Oral Radiol. Endod. 2006; 101: 56-60.

[12] Rawashdeh M. A., Telfah H.: Secondary alveolar bone grafting: the dilemma of donor site selection and morbidity. J. Oral Maxillofac. Surg. 2008; 46: 665-670.

[13] Ozaki W., Buchman S. R.: Volume maintenance of onlay bone grafts in the craniofacial skeleton: micro-architecture versus embryologic origin. Plast. Reconstr. Surg. 1998; 102: 291-299.

[14] Silva R. V., Camili J. A., Bertran C. A., Moreira N. H.: The use of hydroxyapatite and autogenous cancellous bone grafts to repair bone cavities in rats. Int. J. Oral Maxillofac. Surg. 2005; 34: 178-184.

[15] O'Keefe R. M., Reimer B. L., Butterfield S. L.: Harvesting of autogenous cancellous bone graft from the proximal tibial methaphysis: a review of 230 cases. J. Orthop. Trauma 1991; 5: 469-474.

[16] Alt V., Nawab A., Seligson D.: Bone grafting from the proximal tibia. Trauma 1999; 47: 555-557.

[17] Ilankovan V., Stronczek M., Telfer M., Peterson L. J., Stassen L. F. A., Ward-Booth P.: A prospective study of trephined bone grafts of the tibial shaft and iliac crest. Br. J. Oral Maxillofac. Surg. 1998; 36:434-439.

[18] Hashemi H. M.: Oblique use of a trephine bur for the harvesting of tibial bone grafts. Br. J. Oral Maxillofac. Surg. 2008; 46: 690-691.

[19] Belsy W., Ward-Booth P.: Technique for harvesting tibial cancellous bone modified for use in children. Br. J. Oral Maxillofac. Surg. 1999; 37: 129-133.

[20] Hughes C. W., Revington P. J.: The proximal tibia donor site in cleft alveolar bone grafting: experience of 75 consecutive cases. J. Cranio Maxillofac. Surg. 2002; 30: 12-16.

[21] Chen Y. C., Chen C. H., Chen P. L., Huang I. Y., Shen Y. S., Chen C. M.: Donor site morbidity after harvesting of proximal tibia bone. Head Neck 2006; 28: 496-500. 
[22] Catone G. A., Reimer B. L., McNeir D., Ray R.: Tibial autogenous cancellous bone as an alternative donor site in maxillofacial surgery: a preliminary report. J. Oral Maxillofac Surg. 1992; 50: 1258-1263.

[23] Thor A., Farzad P., Larsson S.: Fracture of the tibia: Complication of bone grafting to the anterior maxilla. Br. J. Oral Maxillofac. Surg. 2006; 44: 46-48.

[24] Kalaaji A., Lilja J., Elander A., Friede H.: Tibia as donor site for alveolar bone grafting in patients with cleft lip and palate: long-term experience. Scand. J. Plast. Reconstr. Surg. Hand Surg. 2001; 35: 35-42.

[25] Walker T. M. W., Modayil P. C., Cascarini L., Williams L., Duncan S. M., Ward-Booth P.: Retrospective review of donor site complications after harvest of cancellous bone from the anteriomedial tibia. 2009; 47: 20-22. 